\title{
Dialog in der Sozialen Arbeit mit Familien
}

\section{Einleitung in den Schwerpunkt}

Über Dialog wird im Zusammenhang Sozialer Arbeit spätestens seit Ende der 60 er Jahre gesprochen. Damals ging es vordergründig um die Beseitigung von Hierarchien und überkommener Herrschaftsstrukturen in der Gesellschaft und in der Sozialen Arbeit. Es ging allerdings auch darum, zwischen unterschiedlichen gesellschaftlichen Schichten anders als bis dahin ins Gespräch zu kommen.

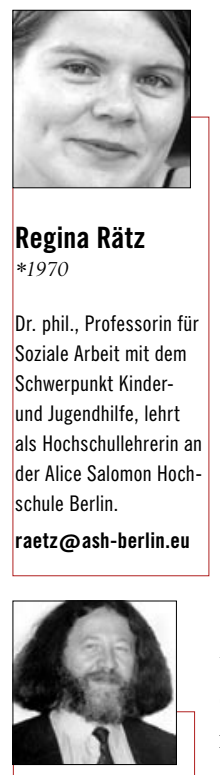

Inzwischen - so ist die Annahme - ist der Dialog als Inhalt und Anspruch Sozialer Arbeit angekommen. Das gilt insbesondere im Hinblick auf die rechtlichen Grundlagen der Beteiligung und Aushandlung, etwa im SGB VIII, sowie auf neu entwickelte Methoden in der Sozialen Arbeit mit Familien. Dass sich dialogische Methoden und Arbeitsansätze etablieren konnten, ist im Zusammenhang mit der sich verstärken-

Hans-Ullrich Krause $* 1954$ Vorsitzender der Internationalen Gesellschaft für erzieherische Hilfen (Frankfurt/ M), Sprecher Regionalgruppe Berlin, Kinderhaus Berlin Mark Brandenburg, Kronberger Kreis für Qualitätsentwicklung.

krause@ Kinderhaus-b-b.de den Demokratisierung der Gesellschaft und der Sozialen Arbeit zu verstehen.

Fachkräfte, die mit einer demokratischen Grundhaltung und entsprechendem Habitus ihre Praxis gestalten, erleben ganz direkt, dass sich Dialog wirklich auszahlt. Wenn sich Kinder, Jugendliche, Eltern beteiligt fühlen, ist der Erfolg einer Hilfe in aller Regel wahrscheinlicher: einbezogene Menschen erleben den Diskurs auf Augenhöhe und sind nicht zuletzt daher eher bereit, Verantwortung für sich und andere zu übernehmen. Sie können sich besser selbst verstehen, können sich im Kontext zu anderen begreifen, können den Willen entwickeln, die Probleme in ihren Ursachen und Ursprüngen zu ergründen und positiv zu wenden.

Doch es sind nicht allein die unmittelbaren Arbeitszusammenhänge in der Sozialen Arbeit, wie z.B. der Hilfeprozess, von Interesse, wenn es um Dialog geht. Es sind auch die Organisationen selbst, die sich insbesondere dann erfolgreich weiterentwickeln können, wenn die Beteiligten im Dialog miteinander sind und bleiben. Moderne Organisationen haben erkannt, dass es ohne Dialog nicht geht.

Allerdings gibt es auch gegenläufige Tendenzen. Diese drücken sich gesellschaftlich z.B. in den großen und kleinen Konfliktthemen aus, wobei unterschiedliche Interessenkonstrukte immer härter aufeinander treffen, wie man am Beispiel des Stuttgarter Hauptbahnhofs sehen kann. Auch in der Sozialen Arbeit gibt es Entwicklungen, die das Dialogprinzip außer Kraft setzen und begonnene Entwicklungen des Aufeinanderzugehens wieder in Frage stellen. Deutlich wurde das insbesondere bei den Diskursen über die Novellierung des KJHG/SGB VIII im Hinblick auf den Kinderschutz oder die Entwicklung eines eigenen Kinderschutzgesetzes.

Da wurden plötzlich längst überwunden geglaubte Argumente laut: so wurde u.a. nach mehr Überwachung, mehr Kontrolle, härterem Durchgreifen gerufen. Und dies, obwohl bekannt ist, dass eine $\mathrm{Zu}$ nahme an Kontrolle keine höhere Sicherheit für Kinder bringt und dass eine solidarische, offene und zugewandte Hilfe in aller Regel förderlicher ist. Viel zu wenig beachtet wurde dabei, dass es im Hinblick auf Konflikte im Kinderschutz eher nicht die unzureichende Überwachung seitens der Ämter war, sondern eher die fehlende, ungenaue oder fehlerhafte Kommunikation zwischen Eltern und Jugendamt, zwischen den unterschiedlichen Hilfeorganisationen und entsprechenden Partnerbereichen wie Gesundheit, Polizei, Justiz etc. So war es eben nicht oder eben nur unzureichend zu einem förderlichen Miteinander in der Hilfegestaltung gekommen, ganz zu schweigen von Dialog. Die Behauptung, dass es an den Betroffenen liege, wenn es zu besagten Kommunikationsproblemen kommt, kann entkräftet werden: Immer dann, wenn ein gewollter und professionell abgesicherter bzw. zwischenmenschlich positiver Kontakt entstanden ist, war die Bereitschaft von Eltern, Kindern und Jugendlichen vorhanden, miteinander ins Gespräch zu kommen und zu bleiben. Das können ExpertInnen sowohl im Bundesmodellprojekt „Aus Fehlern lernen“ als auch bei dem zweijährigen Bundesforschungsprojekt „Sozialpädagogische Familiendiagnosen in Kinderschutzfällen“ belegen.

Es ist also notwendig, weiter über Dialog in der Sozialen Arbeit nachzudenken und zu diskutieren. Zum einen, weil es wichtig ist, die positiven Erfahrungen aus unterschiedlichen Arbeitszusammenhängen zur Kenntnis zu nehmen und diese kritisch zu hinterfragen; zum anderen, um sich konservativen, wenig hilfreichen Gegenbewegungen mit fachlichen und fachpolitischen Argumenten in den Weg stellen zu können. Die Beiträge dieses Schwerpunkts berichten aus der Sozialen Arbeit mit Familien: von den Möglichkeiten dialogischer Praxis, von eigenen Erfahrungen der AutorInnen mit dialogischen Ansätzen, aber auch von Hemmnissen, blinden Flecken und gesellschaftlichen Wandlungsprozessen, die Dialogverfahren erschweren. 